\title{
Structural Alteration of Erythrocyte Membrane during Storage: a Combined Electrical Conductometric and Flow-Cytometric Study
}

\author{
Federico Bordi ${ }^{\mathrm{a}}$, Cesare Cametti ${ }^{\mathrm{b} *}$, Francesco De Luca ${ }^{\mathrm{b}}$, Tommaso Gili ${ }^{\mathrm{b}}$, Roberta \\ Misasi $^{c}$, Maurizio Sorice ${ }^{c}$, Annapia Circella ${ }^{c}$ and Tina Garofalo ${ }^{c}$ \\ a Dipartimento di Medicina Interna, Universita' di Roma "Tor Vergata", Rome, Italy and \\ Istituto Nazionale per la Fisica della Materia (INFM), Unita' di Roma1 \\ b Dipartimento di Fisica, Universita' di Roma "La Sapienza" Piazzale A. Moro 5 - \\ I-00185 Rome, Italy and Istituto Nazionale per la Fisica della Materia (INFM), \\ Unita' di Roma1. Fax: +39064463158; E-mail: cesare.cametti@roma1.infn.it \\ c Dipartimento di Medicina Sperimentale e Patologia, Universita' di Roma "La Sapienza", \\ Rome, Italy \\ * Author for correspondence and reprint requests \\ Z. Naturforsch. 56c, 857-864 (2001); received March 21/May 9, 2001 \\ Erythrocytes, Dielectric Measurements, Flow-Cytometric Measurements \\ Alterations in the electrical passive parameters of red blood cell membranes occurring during \\ storage have been investigated by means of two different experimental approaches, i.e., ra- \\ diowave dielectric spectroscopy measurements and flow-cytometric measurements. We ob- \\ served a correlation between the appearance of phosphatidylserine molecules in the outer \\ leaflet of the cell membrane and the occurrence of a change in the electrical passive mem- \\ brane parameters. The electrical re-organization of the membrane, resulting in an increase \\ of its conductivity and permittivity after 5-7 days from blood storage, can be considered \\ as a precursory event for the loss of asymmetry in the lipid distribution across red blood \\ cell membrane.
}

\section{Introduction}

The lipid topology of biological cell membranes, particularly of eukaryotic cells, has been investigated extensively and several comprehensive reviews on this subject have appeared in the last few years (Devaux, 1992; Zachowski, 1993). In particular, it has been shown that in red blood cells under physiological conditions, phosphatidylserine (PS) is localized exclusively in the inner leaflet of the plasma membrane (Gupta, 1992) and this transmembrane asymmetry is an essential feature involved in macrophage recognition and splenic clearance of aged or abnormal erythrocytes (Schroit et al., 1985; McEvoy et al., 1986). This asymmetry results from a balance of two different mechanisms, a passive diffusion across the bilayer and an active transport maintained by aminophospholipid translocase which continuously pumps PS (and PE) from the outer to the inner leaflet of the membrane (Schroit and Zwaal, 1991).

The loss of asymmetry of endogenous phosphatidylserine becomes apparent only when the aminophospholipid tranlocase activity is impaired.
In particular, two proteins have been proposed as candidate transporters, i.e., a PS-binding, sulfhydryl-containing erythrocyte protein, with $32 \mathrm{kD}$ molecular weight (Connor and Schroit, 1989) and a $\mathrm{Mg}^{2+}$-ATPase of $110-120 \mathrm{kD}$ molecular weight (Zimmermann and Daleke, 1993). In the PS translocation, the involvement of cytoskeleton proteins remains controversial. In the past, several authors (Haest et al., 1978; Raval and Allan, 1984) have suggested that selective interactions between aminophospholipids and cytoskeleton spectrin protein could occur and participate to the overall dynamic characterization of phospholipids in the erythrocyte membrane. More recently, however, experiments carried out with cytoskeleton depleted red cells (Beleznay et al., 1993), with spectrin-deficient red cells (Kuypers et al., 1993) and with heat-denaturated cytoskeleton proteins (Loh and Huestis, 1993) give support to the hypothesis that cytoskeleton proteins do not play a role in the modulation of lipid asymmetry.

The loss of normal phospholipid asymmetry has been associated with many physiological and pathological phenomena (Westerman et al., 1984), 
including cell death, sickle cell anemia (Kuypers et al., 1994) and aged cells (Kuypers et al., 1996), where the aminophospholipid translocases are severely impaired.

The objective of this work is to evidence alterations in the passive electrical parameters (i.e., the membrane permittivity $\varepsilon^{\prime}$ and the membrane conductivity $\sigma$ ) of the red blood cell membrane occurring during storage and to suggest how these changes, particularly in the membrane conductivity, could play an important role in the control of membrane asymmetry. The appearance of phosphatidylserine (PS) into the outer membrane leaflet of erytrocyte cells, that catalyses the clot formation, could be favoured by an increase of the overall membrane conductivity causing its passive diffusion across the bilayer. The exact mechanism by which phosphatidylserine moves towards the outer monolayer is still not completely understood and the modification in the electrical state of the membrane may be proposed as an initial mediator of this event.

Using two combined techniques such as flow cytofluorimetric measurements and radiowave dielectric spectroscopy measurements we have measured, under certain in vitro conditions, the agedependent changes in the electrical parameters of the cell membrane and the loss of phospholipid asymmetry, particularly the age-dependent increase of phosphatidylserine at the membrane surface.

These two different events occur in different time scales and our investigation suggests that a structural rearrangement of the membrane, as revealed by the increase of the membrane conductivity after about 5-7 days of storage, could favour the phosphatidylserine exposure on the cell surface, that begins to be detectable after 10 days and is highly significant within 20 days of storage.

\section{Experimental}

Human erythrocyte suspensions were prepared from fresh human venous blood obtained from laboratory volunteers. Erythrocytes were pelleted by centrifugation, washed twice with $0.9 \% \mathrm{NaCl}$ and once with incubation buffer, SAG-M buffer $(\mathrm{NaCl} 8.77 \mathrm{~g} / \mathrm{l}$, adenine base $0.211 \mathrm{~g} / \mathrm{l}$, monohydrated glucose $11.25 \mathrm{~g} / \mathrm{l}$, mannitol $6.56 \mathrm{~g} / \mathrm{l}$ ). This buffer does not differ from an alkaline, hypotonic, phosphate-containing solution [adenine, $2 \mathrm{~mm}$; dextrose, $50 \mathrm{~mm}$; mannitol, $20 \mathrm{~mm}$; $\mathrm{NaCl}, 75 \mathrm{~mm}$; $\mathrm{Na}_{2} \mathrm{HPO}_{4}, 9 \mathrm{~mm}$ ] employed by Hess et al. (2000; 2000a) where red blood cells were successfully stored for 9-10 weeks, with in-vivo 24-hour cell recovery fractions greater than 80 per cent and with hemolysis of less than 0.5 per cent. The cell suspensions were finally diluted in the incubation buffer (SAG-M buffer) to the appropriate hematocrit $(\phi=10 \%)$ and were stored at $4{ }^{\circ} \mathrm{C}$.

The exposure of phosphatidylserine (PS) on the surface of stored erythrocytes was evaluated by immunofluorescence and flow cytofluorimetric analysis, using an anti-PS monoclonal antibody (CAL-13, IgG2b,k), with highly restricted binding specificity, since it is not reactive with phosphatidylcholine, sphingomyelin and phosphatidylethanolamine (Ichikawa et al., 1992).

Cells $\left(1 \times 10^{6}\right)$ after washing three times in PBS, were incubated with anti-PS monoclonal antibody (IgG) in PBS/BSA $1 \%$ for $1 \mathrm{~h}$ at $4{ }^{\circ} \mathrm{C}$, followed by addition of fluorescein isothiocyanate (FITC)conjugated goat anti-mouse $\operatorname{IgG}(\gamma$-chain specific, Sigma Chem. Co., St. Louis, (USA)) for $30 \mathrm{~min}$ at $4{ }^{\circ} \mathrm{C}$. After washing with PBS, fluorescence intensity was analyzed by means of a Becton Dickinson cytometer (Becton Dickinson, St. José, CA). Ten thousand events per sample were collected to ensure adequate fluorescence level. The light scatter and fluorescence channels were set on a logarithmic gain scale.

The dielectric and conductometric measurements were carried out in the frequency range from $1 \mathrm{kHz}$ to $1 \mathrm{GHz}$ by means of two HewlettPackard Impedance Analyzers, mod. 4292A (in the frequency interval from $1 \mathrm{kHz}$ to $10 \mathrm{MHz}$ ) and mod. 4191A (in the frequency interval from 1 $\mathrm{MHz}$ to $1 \mathrm{GHz}$ ). The conductivity cell consists of a short section of a coaxial line and the calibration procedure, using standard liquids of known dielectric constant and conductivity has been reported elsewhere (Diociaiuti et al, 1991).

We have followed the time evolution of the stored samples. At different time intervals, appropriate aliquots of cell suspension were withdrawn from the storage bag, immediately before each dielectric and flow-cytofluorimetric measurements. Dielectric measurements were carried out in a period of 13 days and cytofluorimetric measurements for a period of 40 days. During the whole 
storage time, erythrocyte hemolysis, measured by analyzing the hemoglobin content in the supernatant, was negligible (less than $1 \%$ ).

The influence of a specific inhibitor on the aminophospholipid translocase was also studied. We use N-ethyl-maleimide (NEM) that inhibits the aminophospholipid translocase by complexing a sulfhydryl group involved in the PS transport (Kuypers et al., 1993; Zachowski, 1993). Two different erythrocyte samples with a different treatment were investigated. In the former, erythrocyte cells at an appropriate hematocrit $(\varnothing=10 \%)$ were incubated for $30 \mathrm{~min}$ at room temperature in a buffer containing NEM (Sigma) $10 \mathrm{~mm}$. Subsequently, the cells were washed in SAG-M buffer (without NEM) and then stored at $4{ }^{\circ} \mathrm{C}$. Alternatively, erythrocyte cells at $\varnothing=10 \%$ were incubated for $30 \mathrm{~min}$ at room temperature in a SAG-M buffer containing NEM $10 \mathrm{~mm}$ and then stored directly in the same buffer. For both the samples, dielectric and conductometric measurements were taken each day for a period of 10 days.

Finally, erythrocyte cells were directly stained with FITC-coniugated annexin V (Bender MedSystems Diagnostics GmbH, Wien, Austria). After washing with PBS at $4{ }^{\circ} \mathrm{C}$, fluorescence intensity was analyzed by a Coulter Epics flow cytometer (Coulter, Hialeah, FL).

\section{Dielectric and conductometric measurements}

The dielectric model of an erythrocyte cell

An erythrocyte cell in an aqueous solution can be modelled, from an electrical point of view, as an oblate spheroid (the cytosol) characterized by a complex conductivity

$$
\sigma_{p}^{*}(\omega)=\sigma_{p}+i \omega \varepsilon_{0} \varepsilon_{p}
$$

covered by another confocal ellipsoid of thickness $\delta$ (the cytoplasmatic membrane) of complex conductivity

$$
\sigma_{s}^{*}(\omega)=\sigma_{s}+i \omega \varepsilon_{0} \varepsilon_{s}
$$

and dispersed in a continuous medium (the extracellular medium) of complex conductivity

$$
\sigma_{m}^{*}(\omega)=\sigma_{m}+i \omega \varepsilon_{0} \varepsilon_{m}
$$

This scheme is common in the study of the dielectric properties of heterogeneous systems. As far as red blood cell suspensions are concerned, the details are elsewhere (Bordi et al., 1997). Briefly, un- der the influence of an (time-dependent) electric field, each cell experiences the presence of an induced dipole moment resulting from the different electrical properties of the different media disjoined by the cell membrane. The overall result of this complex behaviour of the cell suspension is the appearance of a well-defined dielectric and conductometric dispersion (known as the $\beta$-dispersion, already called Maxwell-Wagner dispersion) generally occurring in the radiowave frequency range whose characteristics (the dispersion strength and the relaxation time) depend on the aqueous phase-membrane interface.

Within the effective medium theory approximation (Foster and Schwan, 1986), the membrane parameters (the permittivity $\varepsilon_{s}$ and the conductivity $\sigma_{s}$ and the conductivity of the cytosol $\sigma_{p}$ ) can be properly evaluated.

\section{Analysis of the dielectric spectra}

A typical dielectric spectra (the permittivity $\varepsilon^{6}$ and the electrical conductivity $\sigma$ ) in the frequency range from $1 \mathrm{kHz}$ to $1 \mathrm{GHz}$ is shown in Fig. 1 . As can be seen, the Maxwell-Wagner dispersion, occurring between $200 \mathrm{kHz}$ and $100 \mathrm{MHz}$, is located in between two other dispersions, the first at low frequency, due to the electrode polarization, and the second, at high frequencies, due to the orientational polarization of the water molecules. These two contributions must be properly taken into account in the procedure performed to extract the correct values concerning the interfacial polarization of the erythrocyte suspension.

As far as the high frequency dispersion is concerned, the effective medium theory can be modified by adding the contribution due to the dielectric dispersion of the aqueous phase, as described by a Debye-type relaxation function according to

$$
\varepsilon^{6}{ }_{H_{2} \mathrm{O}}(\omega)-i \varepsilon^{\prime}{ }_{\mathrm{H}_{2} \mathrm{O}}(\omega)=\varepsilon_{\mathrm{H}_{2} \mathrm{O}}+\frac{(\Delta \varepsilon)_{\mathrm{H}_{2} \mathrm{O}}}{1+i \omega \tau_{\mathrm{H}_{2} \mathrm{O}}}
$$

The dielectric parameters of the aqueous phase, $(\Delta \varepsilon)_{H_{2} \mathrm{O}}, \varepsilon_{\mathrm{H}_{2} \mathrm{O}}$ and $\tau_{\mathrm{H}_{2} \mathrm{O}}$ as a function of temperature are given elsewhere (Hasted, 1973).

At room temperature, the relaxation time of the water is $\tau_{H_{2} \mathrm{O}}=910^{-12} \mathrm{~s}$, which corresponds to a relaxation frequency of $v_{\mathrm{H}_{2} \mathrm{O}}=17 \mathrm{GHz}$. Consequently, this dispersion extends besides the frequency range investigated in the present work and 

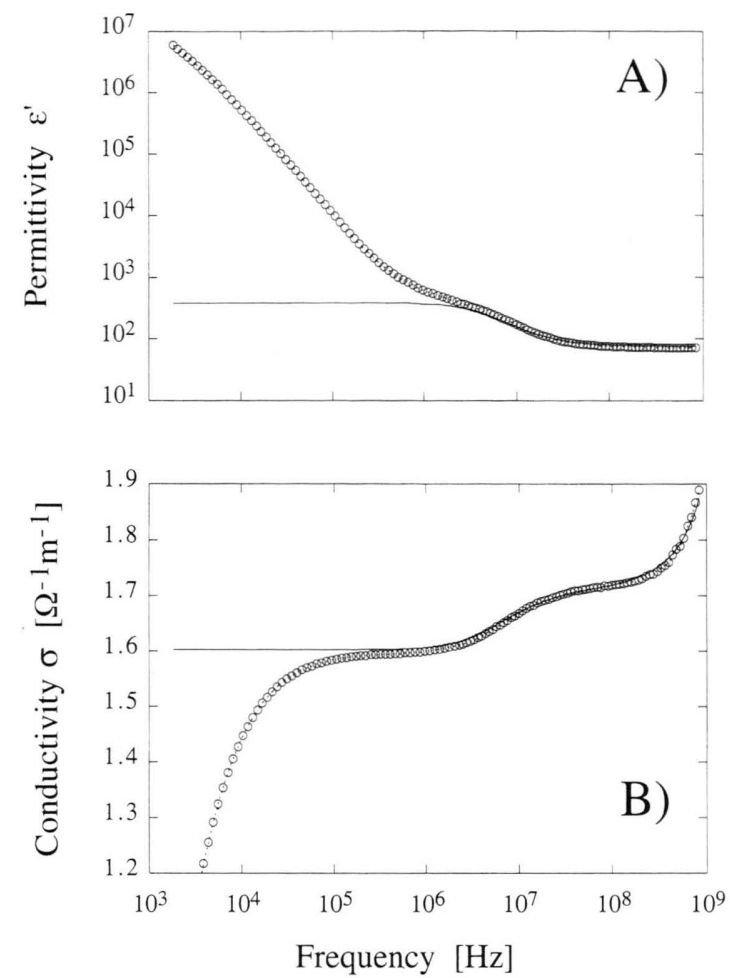

Fig. 1. Dielectric and conductometric spectra of red blood cell suspensions in the frequency range from 1 $\mathrm{kHz}$ to $1 \mathrm{GHz}$. A): the permittivity $\varepsilon^{*} ; \mathrm{B}$ ): the electrical conductivity $\sigma$. The Maxwell-Wagner dispersion occurs in the frequency range from $200 \mathrm{kHz}$ to $100 \mathrm{MHz}$. The electrode polarization effect (in the low frequency range) and the polarization of the aqueous phase (in the high frequency range) are also shown. The full lines represent the calculated values of the Maxwell-Wagner dispersion on the basis of the effective medium theory. The values of the electrical membrane parameters are shown in Figs. 3 and 4.

we can observe only its low-frequency tail. However, as can be seen in Fig. 1, the addition of this contribution is able to completely describe the observed behaviour both in the permittivity $\varepsilon^{6}$ and in the conductivity $\sigma$ (see, for example, the increase in the conductivity $\sigma$ between $500 \mathrm{MHz}$ and $1 \mathrm{GHz}$ ).

As far as the electrode polarization effect is concerned, we observe the typical increase, at frequencies lower than $100 \mathrm{kHz}$, of the imaginary part of the complex impedance, resulting in the high values in the permittivity $\varepsilon^{6}$. This behaviour can be taken into account if a impedance contribution governed by a power-law

$$
Z_{p o l}=A \omega^{\alpha}
$$

is added to the measured impedance of the whole sample under investigation. The parameters $A$ and $\alpha$ are determined for each sample and for each temperature investigated following the procedure suggested by Raico et al. (Raico et al., 1998). As can be seen in Fig. 1, the correction takes into account the electrode polarization contribution and allows to extract the "true" low-frequency values of the Maxwell-Wagner dispersion.

An important test of consistency of the whole procedure we have employed in the analysis of the dielectric data is that the same set of parameters are able to describe both the permittivity $\varepsilon^{b}$ and the conductivity $\sigma$ over the whole frequency range we have investigated.

\section{Flow-cytofluorimetric measurements}

Incubation of red blood cells with anti-PS monoclonal antibody ( $\mathrm{IgG}$ ) used in flow cytometry offers an appropriate method for the determination of loss of normal phospholipid asymmetry. Fig. 2 shows the flow cytometric analysis of red blood cells at different storage times up to 40 days
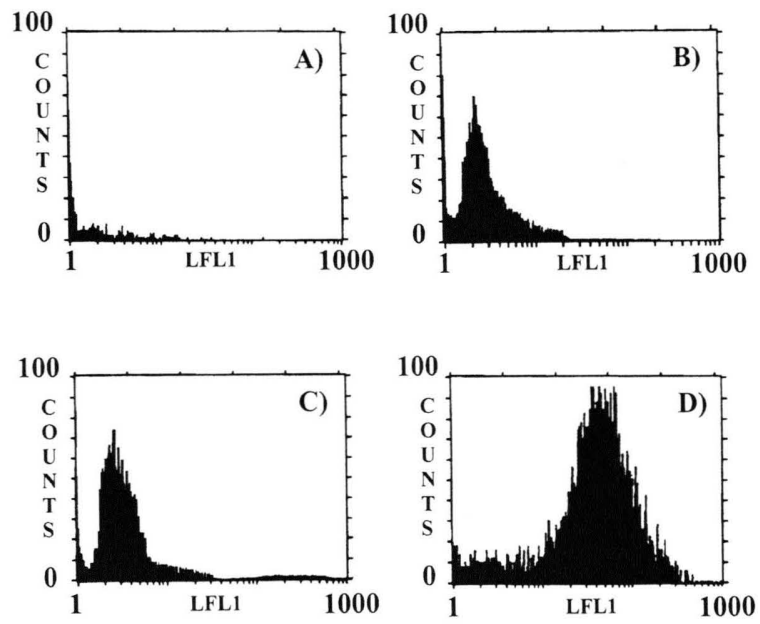

Fig. 2. Flow cytometric analysis of the reactivity of erythrocyte cells at different storage time. Cells were stained with anti-PS monoclonal antibody, followed by incubation with FITC-conjugated goat anti-mouse IgG. A): fresh erythrocytes, mean fluorescence intensity [MFI] = 5.00 ; B): erythrocytes stored for 10 days, $[\mathrm{MFI}]=11.18$; C): erythrocytes stored for 20 days, $[\mathrm{MFI}]=27.98 ; \mathrm{D})$ : erythrocytes stored for 30 days, $[\mathrm{MFI}]=49.71$. Histograms represent cell number vs. log fluorescence, gated on erythrocyte population of a side scatter/forward scatter histogram. The fluorescence intensity is plotted in three logarithmic decades on the $x$-axis. 
from the initial preparation of cell suspension. In the figure, histograms represent log fluorescence versus cell number, gated on erythrocyte population of a side scatter/forward scatter histogram. Cell number is indicated on the $y$-axis and fluorescence is represented in three logarithmic units at the $x$-axis.

\section{Results and Discussion}

Our results show a progressive increase of phosphatidylserine (PS) staining upon storage time, which demonstrates an exposure of PS staining on the surface of erythrocyte cells. We investigated this phenomenon within 40 days of storage and observed that anti-PS $\mathrm{mAB}$ binds to the surface of aged cells. The cytofluorimetric analysis showed a time-dependent binding of the antibody to cell surface, as revealed by the increase of the mean fluorescence intensity (MFI) (see Fig. 2). The increase in cell membrane binding was highly significant after 10 days (MFI $=11.2$, against a value of about 5.0 at the initial day, $p=0.001$ ) and was more evident after longer incubation. The same binding was observed using FITC-conjugated annexin V (data not shown), indicating the time-dependent increase of PS expression on the surface of erythrocyte cells. The mean fluorescence intensity, proportional to the amount of PS located in the outer membrane layer, is shown in Fig. 3, for both the inhibitor NEM and the protein annexin V.

The electrical behaviour of the cell membrane is summarized in Figs. 4 and 5, where the electrical parameters $\sigma_{s}, \varepsilon_{s}$ and $\varepsilon_{p}$ are shown as a function of storage time, at three different temperatures.

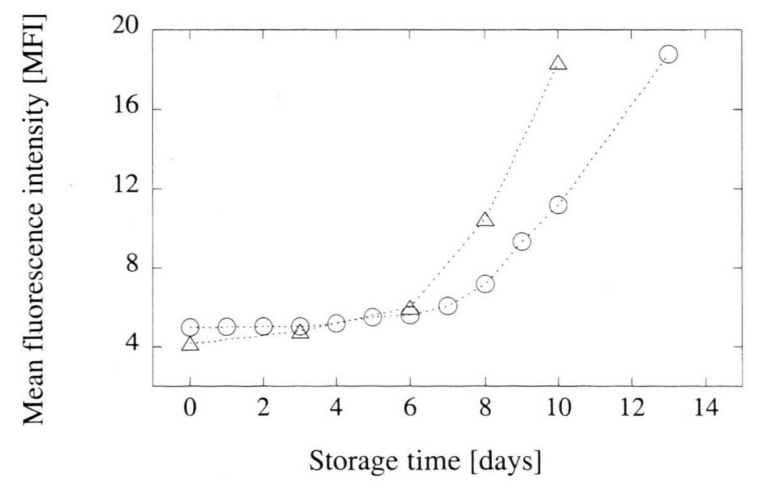

Fig. 3. The mean fluorescence intensity as a function of storage time. $(\bigcirc)$ : NEM; $(\triangle)$ : annexin V.

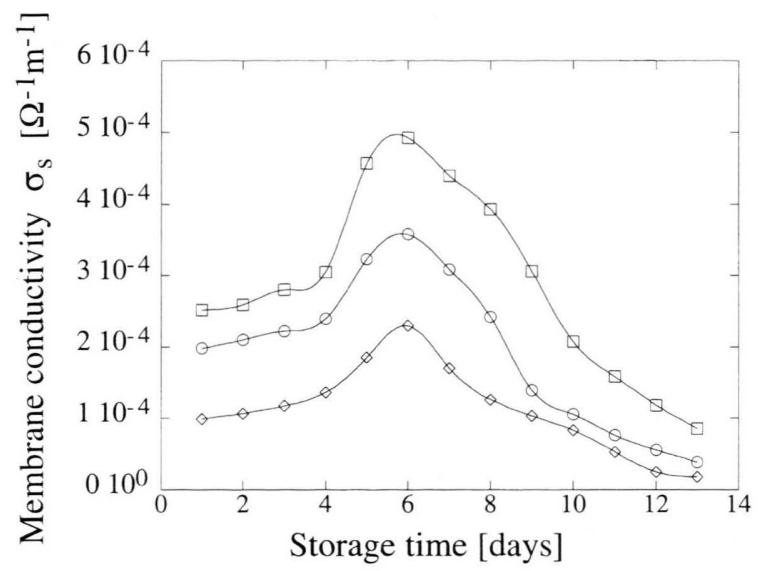

Fig. 4. The membrane conductivity $\sigma_{\mathrm{s}}$ as a function of storage time at different temperatures: $(\diamond): T=4{ }^{\circ} \mathrm{C}$; (○): $T=24{ }^{\circ} \mathrm{C} ;(\square): T=37^{\circ} \mathrm{C}$. The full lines are the cubic spline fit of the data and serve to guide eye only.
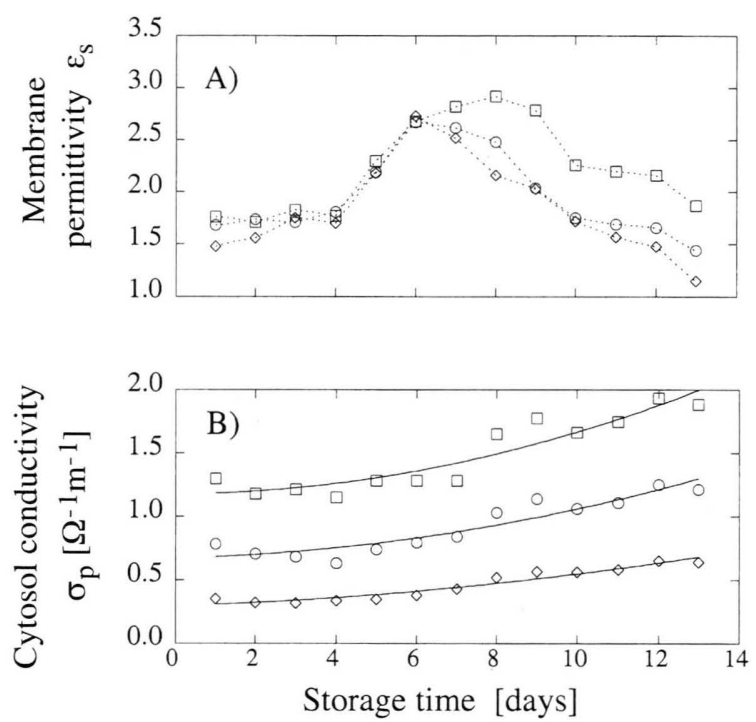

Fig. 5. The membrane permittivity $\varepsilon_{\mathrm{s}}$ (A) and the cytosolconductivity $\sigma_{\mathrm{p}}(\mathrm{B})$ of red blood cell membrane as a functionof storage time at three different temperatures: $(\diamond): T=4{ }^{\circ} \mathrm{C} ;(\bigcirc): T=24^{\circ} \mathrm{C} ;(\square): T=37^{\circ} \mathrm{C}$.

The main feature is a marked increase of the membrane conductivity and membrane permittivity at 5-7 days from the initial storage, followed by a more or less regular decrease toward the initial values, at later time. This finding must be compared with the flow-cytofluorimetric measurements (Figs. 2 and 3) which display a progressive increase of the PS exposure as a function of time, although its maximum concentration occurs at 
about 30-40 days from the beginning of the storage. If there is a correlation between the two effects, the alteration in the membrane conductivity (an increase in the transport across the membrane) and in the membrane permittivity (a different charge and or polar groups arrangement) could be considered as a precursory phenomenon that facilitates the phospholipid (PS) translocase across the cell membrane.

A strong support to a correlation between the two effects comes from measurements carried out in the presence of an inhibitor of the aminophospholipid translocase. Fig. 6 shows the membrane conductivity as a function of time when the erythrocyte cells are incubated with $\mathrm{N}$-ethyl-maleimide (NEM) inhibitor, with two different procedures. If the incubation occurs in a limited period of time and successively the inhibitor is removed from the sample investigated, we observe an increase, although smaller, in membrane conductivity. On the contrary, if the presence of the inhibitor is maintained during all the storage time, the membrane conductivity does not change with time and maintains its initial value during the observation time interval (up to 10 days). At the same time, the expression of phosphatidylserine on the outer membrane leaflet is strongly reduced and practically disappears. These findings suggest that the change in the electrical parameters of the cell

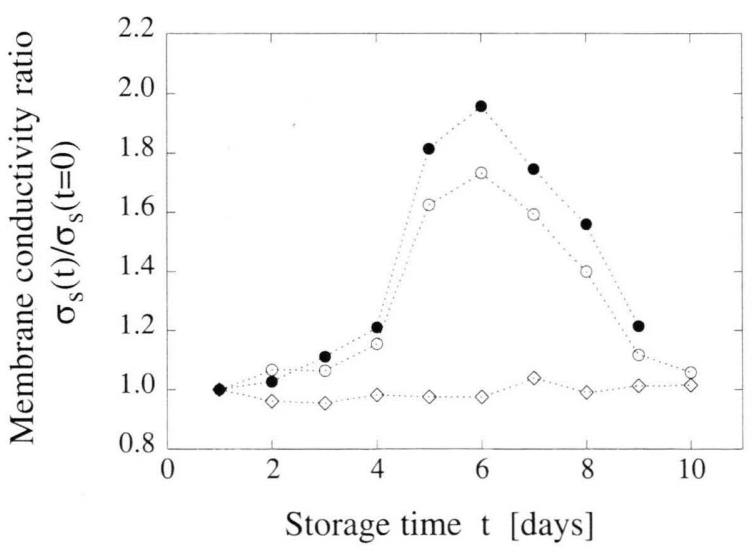

Fig. 6. The membrane conductivity $\sigma_{\mathrm{s}}$ as a function of storage time normalized to the initial value $(t=0)$ of the conductivity, for three different erythrocyte suspensions $(\bullet)$ : cells stored without inhibitor; $(\bigcirc)$ : cells incubated with NEM for $30 \mathrm{~min}$ and subsequently washed and stored in SAG-M buffer solution; $(\diamond)$ : cells incubated with NEM for $30 \mathrm{~min}$ and stored in the presence of the inhibitor. membrane and the occurrence of phospholipid asymmetry in blood red cells are correlated and that the first one could be a necessary and precursory event for the second and more biologically relevant one.

Notheworthy both membrane permittivity $\varepsilon_{\mathrm{s}}$ and membrane conductivity $\sigma_{\mathrm{s}}$ show a remarkable increase at about 6-7 days after the initial stora ge of the cell suspension, the conductivity $\sigma_{\mathrm{p}}$ of the cytosol, however, does not show any marked changes at the same time, but it undergoes only a weak continuous increase as a function of time. This means that the overall ionic transport across the membrane remains approximately unchanged (there is only a small increase upon time) and, above all, that the observed modifications in the membrane permittivity and membrane conductivity relate to the membrane itself, rather than to an alteration of the ionic fluxes toward the intracellular medium.

Although the dielectric cell model we have employed (single shell model) cannot discriminate between the outer and inner monolayer of the membrane bilayer, nevertheless, the pronounced changes in both the membrane permittivity $\varepsilon_{s}$ and conductivity $\sigma_{s}$ suggests that a functional and structural modification in the whole membrane takes place, favouring the PS exposure at the membrane surface with a consequent reduction of the phospholipid asymmetry.

Biological consequences of phospholipid asymmetry have been discussed in detail by Devaux and Zachowski (1994). In particular, in red bood cells (Tait and Gibson, 1994) or platelets (Gaffet et al., 1994) storage, the externally exposure of phosphatidylserine increases steadily, the phenomenon being dramatically relevant after ten days of storage. Interestingly, it has been suggested that reorientation of phosphatidylserine may be one of the recognition mechanisms in the circulation. This regulation plays an important role in membrane-linked enzyme function, in blood coagulation, in modulation of the macrophage immune response and in various cell-cell interactions, including macrophage recognition of aged erythrocytes and apoptotic or cancer cells or adherence of circulating cells to endothelial cells.

It should be noted that even limited level of hemolysis will greatly interfere with the interpretation of the data because in the dielectric analysis, 
the cell concentration must be exactly known and non-intact or partially open cell structures do not contribute to the dielectric dispersion. As far as the hemolysis is concerned, we have found that this effect is small and confined within less than $1 \%$ of the suspended cells and these levels are unable to cause the observed changes in the electrical membrane parameters.
Nevertheless the dielectric methods probe the whole cell suspension and give only average values, they are an useful and non-invasive tool to investigate the overall electrical properties of biological systems, particularly biological cell suspensions.
Beleznay, Z., Zachowski A., Devaux P. F., Navazo M. P. and Ott P. (1993), ATP-dependent aminophospholipid translocation in erythrocyte vesicles: stoichiometry of transport. Biochemistry 32, 3146-3152.

Bordi F., Cametti C., Misasi R., De Persio R. and Zimatore G. (1997), Conductometric properties of human erythrocyte membrane: dependence on hematocrit and alkali metal ions of suspending medium. Eur. Biophys. J. 26, 215-225.

Connor J. and Schroit A. J. (1989), Transbilayer movement of phosphatidylserine in erythrocytes: inhibition of transport and preferential labelling of a 31000-dalton protein by sulfhydrylreactive reagents. Biochemistry 28, 9680-9685.

Devaux P. F. (1992), Protein involvement in transmembrane lipid asymmetry. Annu. Rev. Biophys. Biomol. Struct. 21, 417-439.

Devaux P. F. and Zachowski A. (1994), Maintenance and consequences of membrane phospholipid asymmetry. Chem. Phys. Lipids 73, 107-120.

Diociaiuti M., Molinari A., Calcabrini A., Arancia G., Isacchi F., Bordi C. and Cametti C. (1991), Alteration of passive electrical properties of adriamicyn-treated red cell membranes deduced from dielectric spectroscopy. Bioelectrochem. Bioenerg. 26, 177-184.

Foster K. R. and Schwan H. P. (1986), CRC Handbook of Biological Effects of Electromagnetic Fields (C. Polk and A. Postow Eds.). CRC Press, Boca Raton, FL.

Gaffet P., Basse' F. and Bievenue A. (1994), Loss of phospholipid asymmetry in human platelet plasma membrane after 1-12 days of storage. Eur. Biophys. J. 22, $1033-1040$.

Gupta C. M. (1992), Genesis and maintenance of erythrocyte membrane phospholipid asymmetry: comparative role of aminophospholipid pump and membrane skeleton-bilayer interaction. In: Biomembrane Structure and Functions. The State of Arts (B. P. Gaber and K. R. K. Easwaran, eds.) Adenine Press.

Haest C. W. M., Plasa G., Kamp D. and Deuticke B. (1978), Spectrin as a stabilizer of phosholipid asymmetry in human erythrocyte membranes. Biochim. Biophys. Acta 509, 21-32.

Hasted J. B. (1973), Aqueous Dielectrics. Chapman and Hall, London.
Hess J. R., Rugg N., Knapp A. D., Gormas J. F., Silberstein E. B. and Greenwalt T. J. (2000), Successful storage of RBC's for 10 weeks in a new additive solution. Transfusion 40,1012-1016.

Hess J. R., Rugg N., Knapp A. D., Gormas J. F., Silberstein E. B. and Greenwalt T. J. (2000a), Successful storage of RBC's for 9 weeks in a new additive solution. Transfusion 40, 1007-1011.

Ichikawa K., Suzuki T., Hashimoto Y., Sumida T., Tomioka H., Yoshida S. and Koike T. (1992), Monoclonal autoantibodies to cardiolipin derived from SLE mice. Lupus 1, 239-247.

Kuypers F. A., Lubin B. H., Yee M., Agre P., Devaux P.F. and Geldwerth (1993), The distribution of erythrocyte phospholipids in hereditary spherocytosis demonstrates a minimal role for erythrocyte spectrin on phospholipid diffusion and asymmetry. Blood 81, 1051-1057.

Kuypers F. A., van der Berg J. J. M. and Lubin B. H. (1994), Phospholipid asymmetry and diffusion in the membrane of normal and sickle erythrocytes. In: Membrane Linked Disease (S. Onishi, Ed.). Boca Raton, FL, CRC Press, pp. 21-45.

Kuypers F. A., Lewis R. A., Hua M., Schott M. A., Discher D. and Ernst J. D. (1996), Detection of altered membrane phospholipid asymmetry in subpopulations of human red blood cells using fluorescently labeled annexin V. Blood 87, 1179-1187.

Loh R. K. and Huestis W. H. (1993), Human erythrocyte membrane lipid asymmetry: transbilayer distribution of rapidly diffusing phosphatidylserine. Biochemistry 32, $11722-11726$.

McEvoy L., Williamson P. and Schiegel R. A. (1986), Membrane phospholipid asymmetry as a determinant of erythrocyte recognition by macrophages. Proc. Natl. Acad. Sci. USA 83, 3311-3315.

Raico V., Saibara T. and Irimajiri A. (1998), Dielectric properties of rat liver in vivo: a non invasive approach using an open-ended coaxial probe at audio/radio frequencies. Bioelectrochem. Bioenerg. 47, 325-332.

Raval P. J. and Allan D. (1984), Phospholipid asymmetry in the membrane of intact human erythrocytes and in spectrin-free microvesicles derived from them. Biochim. Biophys. Acta 772, 192-196.

Schroit A. J., Madsen J. W. and Tanaka Y. (1985), In vivo 
recognition and clearance of red blood cells containing phosphatidylserine intheir plasma membranes. J. Biol. Chem. 260, 5131-5138.

Schroit A. J. and Zwaal (1991), Transbilayer movement of phospholipids in red cell and platelet membranes. Biochim. Biophys. Acta 1071, 313-329.

Tait J. F. and Gibson D. (1994), Measurement of membrane phospholipid asymmetry in normal and sicklecell erythrocytes by means of annexin $\mathrm{V}$ binding. J. Lab. Clin. Med. 123, 741-748.
Westerman M. P., Cole E. R. and Wu K. (1984), The effect of spicules obtained from sickle red cells on clotting activity. Br. J. Haematol. 56, 557-562.

Zachowski A. (1993), Phospholipids in animal eukaryotic membranes: transverse asymmetry and movement. Biochem. J. 294, 1-14.

Zimmerman M. L. and Daleke D. L. (1993), Regulation of a candidate aminophospholipid-transporting ATPase by lipids. Biochemistry 32, 12257-12263. 